\title{
Symmetry and decoherence-free subspaces in quantum neural networks
}

\author{
Altaisky M.V., ${ }^{1, *}$ N. E. Kaputkina, ${ }^{2}$ and V. A. Krylov ${ }^{3}$ \\ ${ }^{1}$ Space Research Institute RAS, Profsoyuznaya 84/32, Moscow, 117997, Russia \\ ${ }^{2}$ National Technological University "MISIS", \\ Leninsky prospect 4, Moscow, 119049, Russid \\ ${ }^{3}$ Joint Institute for Nuclear Research, \\ Joliot Curie 6, Dubna, 141980, Russid ț
}

\begin{abstract}
Evolution of quantum states of array of quantum dots is analyzed by means of numerical solution of the von Neumann equation. For two qubit system with dipoledipole interaction and common phonon bath the evolution of the symmetric state $\frac{\uparrow \downarrow+\downarrow \uparrow}{\sqrt{2}}$ leads to the mixture of the triplet states, leaving the singlet decoupled. For three qubit system $\left(D_{1 / 2}^{\otimes 3}=D_{3 / 2}+2 D_{1 / 2}\right)$ with common phonon bath we observed similar effects within the quartet state $D_{3 / 2}$ if all qubits were symmetrically connected.
\end{abstract}

\section{INTRODUCTION}

Both the increasing data flows of modern computations and the miniaturization of data processing units imply the future high performance computers will be based on some quantum elements, which obey the laws of quantum mechanics. Quantum computational devices, being first proposed by R.Feynman [1], can solve the exponentially hard problems of classical computing in polynomial time [2]. The main obstacle on the way to a scalable quantum computer is the decoherence induced by interaction with environment. This keeps the range of commercially affordable circuit-based computers within a few qubits. The compromise between decoherence and quantum parallelism have been achieved rather recently in a form of adiabatic quantum computers - a quantum neural networks made of SQUIDS [3]. This is not a quantum computer in a strict sense of [2], but a quantum simulator which mimics the

\footnotetext{
* altaisky@rssi.ru

$\dagger$ nataly@misis.ru

$\ddagger$ kryman@jinr.ru
} 
work of the Hopfield network using quantized magnetic fluxes of SQUIDS. Being restricted in the range of problems to be solved, the quantum neural network capacity dramatically increases due to quantum parallelism.

Quantum neural network is a massively parallel processing unit made of a large set of identical quantum elements, interacting to each other by many possible, but controlled ways, capable of a self-organization, called learning [4]. The set of elements may be distinguishable, and hence, directly addressable, as in SQUID network [3], or may be indistinguishable (like the atoms of Bose gas), but still interacting to each other in a controlled way [5]. The learning and the performance rates of a massively parallel quantum processing unit thus depend on its symmetry properties.

\section{QUANTUM NEURAL NETWORKS}

In contrast to visible progress in circuit-based quantum computing [2], the quantum neural network studies were out from the mainstream of quantum information processing until the first hardware implementation of the quantum Hopfield network has been built by D-Wave systems Inc. [6]. The adiabatic quantum computers produced by D-wave systems Inc. are described by the Hamiltonian of the form

$$
H=\sum_{i} K_{i} \sigma_{i}^{x}+\sum_{i} H_{i} \sigma_{i}^{z}+\sum_{i \neq j} J_{i j} \sigma_{i}^{z} \sigma_{j}^{z}
$$

with the "spins" implemented by SQUID elements with two possible directions of magnetic flux, and the problem matrix $J_{i j}$, implemented as a set of inductive couplings between the SQUIDs. The general idea beneath adiabatic quantum computing is to evolve adiabatically the system of identical quantum elements, described by the Hamiltonian of the form

$$
H(t)=\Gamma(t) H_{F}+\Lambda(t) H_{P}, \quad \Gamma(0)=1, \Gamma(\infty)=0, \Lambda(0)=0, \Lambda(\infty)=1,
$$

where at initial time $t=0$ the system is hold in the ground state of fiducial Hamiltonian $H_{F}$, to the ground state of the problem Hamiltonian $H_{P}$ at $t \rightarrow \infty$.

Referring the reader to the original papers on adiabatic quantum computers on SQUIDs [6 8], we would like to emphasize their shortage is a very low operational temperature of about $10^{-1} \mathrm{~K}$ range. This results in a high energy consumption of the cooling system and prevents the construction of portable devices. There is a quest for alternative elements for 
quantum neural networks with the operational temperature higher than that of SQUIDs. (Needless to say that the brain network itself may be a quantum neural network [9, 10].) One of the alternatives promising a quantum neural network operating at room temperature may be quantum dot based quantum neural networks [11 13]. The advantage of quantum dots as elements of quantum neural networks is the flexibility of control of their characteristics, i.e, energy spectra, electron localization radius, interaction strength, by external magnetic field [14, 15].

Regardless particular type of quantum elements, considered as quantum neurons, the obedience of such system to the laws of quantum mechanics assumes the importance of the symmetries of the interaction Hamiltonian. The symmetries affect both the energy spectra and the structure of space of states of quantum system. The individual elements of the network cannot be directly addressed, but only the collective states of the whole network can be controlled.

Hopfield network can either work as minimizer, that search for an optimal configuration $\left(s_{0}, \ldots, s_{N}\right), s_{i}= \pm 1$, that minimize the energy $E(s)=\sum h_{i} s_{i}+\sum_{i \neq j} J_{i j} s_{i} s_{j}$, or as an associative memory, that seeks for an optimal matrix $J_{i j}$ to project an arbitrary input vector $\left(s_{0}, \ldots, s_{N}\right)$ into one of $M \leq N$ possible attractors. For quantum case, specially when all qubits are implemented on SQUIDs or quantum dots, it is impossible to change the elements of the connection matrix continually, say by gradient descent method. In contrast, we can set either $J_{i j}=1$, for connected, or $J_{i j}=0$ for disconnected pairs [6]. The problem of quantum neural network is a discrete optimization problem with respect to either "spins" $s_{i}$, or connection matrix $J_{i j}$, both affected by environment, i.e., the strength of external driving field $K_{i}$, the temperature of the phonon bath, the spectral density of phonons, etc. Driving such system from a specially initial quantum state to the final state with minimal energy is a typical problem for Boltzmann machines. It can be solved by simulated annealing in classical case, or by quantum annealing in quantum case.

In the remaining part of this paper we will study the role of symmetries for a toy model of three-qubit network with dipole-dipole interaction and common phonon bath. 


\section{THREE QUBIT SYSTEM DYNAMICS}

Our toy model consists of $N=3$ identical qubits with two orthogonal quantum states, $|X\rangle$ for eXcited, and $|0\rangle$ for ground state, interacting to each other by means of dipoledipole coupling, and linearly interacting to the common heat bath. It is described by the Hamiltonian:

$H=\sum_{i=0}^{N-1} \frac{\delta_{i}}{2}\left(\sigma_{z}^{(i)}+1\right)+\sum_{i=0}^{N-1} \frac{K_{i}}{2} \sigma_{x}^{(i)}+\sum_{i \neq j} J_{i j} \sigma_{+}^{(i)} \sigma_{-}^{(j)}+\sum_{a, i} g_{a} x_{a}\left|X_{i}\right\rangle\left\langle X_{i}\right|+H_{P h} \equiv H_{0}+H_{I n t}+H_{P h}$,

written in rotating wave approximation, with $\delta_{i}$ being the detuning of the driving electric field frequency from the $i$-th qubit resonance frequency $\frac{E_{X}^{i}-E_{0}^{i}}{\hbar} ; \hbar=1$ assumed hereafter; $K_{i}$ is a coupling to an external driving field, $J_{i j}$ is the dipole-dipole coupling between qubits. The phonon modes $x_{a}$ are assumed to interact only to the excited states $\left|X_{i}\right\rangle[16]$. The pseudo-spin operators of the $i^{\text {th }}$ qubit are:

$$
\sigma_{z}^{(i)}=\left|\mathrm{X}_{i}\right\rangle\left\langle\mathrm{X}_{i}|-| 0_{i}\right\rangle\left\langle 0_{i}\left|, \quad \sigma_{x}^{(i)}=\right| 0_{i}\right\rangle\left\langle\mathrm{X}_{i}|+| \mathrm{X}_{i}\right\rangle\left\langle 0_{i}\left|, \quad \sigma_{+}^{(i)}=\right| \mathrm{X}_{i}\right\rangle\left\langle 0_{i}\left|, \quad \sigma_{-}^{(i)}=\right| 0_{i}\right\rangle\left\langle\mathrm{X}_{i}\right|
$$

Our toy model is an extension of quantum dot interaction model considered in [13].

In case of 3 qubits there are 8 classically distinguished states, from 000 to $X X X$, and also 8 topologically distinguishable types of connection between qubits, $a+3 b+3 c+d$, see Fig. 1. In case of indistinguishable qubits there are only 4 such connection, corresponding to the

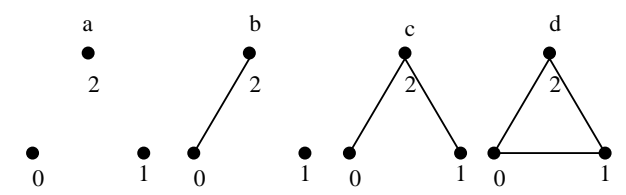

Figure 1. Possible connections in three qubit network

cases a,b,c,d, respectively. Case 'a' is a trivial case of no network, case 'b' corresponds to two qubits considered in our paper [17], so only 'c' and 'd' can be considered as the connections of 3 qubit network. At the absence of interaction with environment, the energy levels of such systems can be easily evaluated. In standard computational basis $(000,00 X, \ldots, X X X)$ the 
free part of the Hamiltonian $H$ can be written as

$$
H_{0}=\left(\begin{array}{cccccccc}
0 & \frac{K_{0}}{2} & \frac{K_{1}}{2} & 0 & \frac{K_{2}}{2} & 0 & 0 & 0 \\
\frac{K_{0}}{2} & 0 & J_{01} & \frac{K_{1}}{2} & J_{02} & \frac{K_{2}}{2} & 0 & 0 \\
\frac{K_{1}}{2} & J_{01} & 0 & \frac{K_{0}}{2} & J_{12} & 0 & \frac{K_{2}}{2} & 0 \\
0 & \frac{K_{1}}{2} & \frac{K_{0}}{2} & 0 & 0 & J_{12} & J_{02} & \frac{K_{2}}{2} \\
\frac{K_{2}}{2} & J_{02} & J_{12} & 0 & 0 & \frac{K_{0}}{2} & \frac{K_{1}}{2} & 0 \\
0 & \frac{K_{2}}{2} & 0 & J_{12} & \frac{K_{0}}{2} & 0 & J_{01} & \frac{K_{1}}{2} \\
0 & 0 & \frac{K_{2}}{2} & J_{02} & \frac{K_{1}}{2} & J_{01} & 0 & \frac{K_{0}}{2} \\
0 & 0 & 0 & \frac{K_{2}}{2} & 0 & \frac{K_{1}}{2} & \frac{K_{0}}{2} & 0
\end{array}\right)
$$

with all detunings set to zero $\delta_{i}=0$. Let us start with the symmetric case 'd'. Setting in (3) all connections equal $J_{02}=J_{01}=J_{12}=J$, and a common driving field $K_{0}=K_{1}=K_{2}=K$ We get the energy eigenvalues

$E_{1,2}=J+\frac{K}{2} \pm \sqrt{K^{2}+J K+J^{2}}, \quad E_{3,4}=J-\frac{K}{2} \pm \sqrt{K^{2}-J K+J^{2}}, \quad E_{5,6,7,8}=-J \pm \frac{K}{2}$.

The last two eigenvalues are doubly degenerated. For less symmetric configuration of $\Lambda$ -

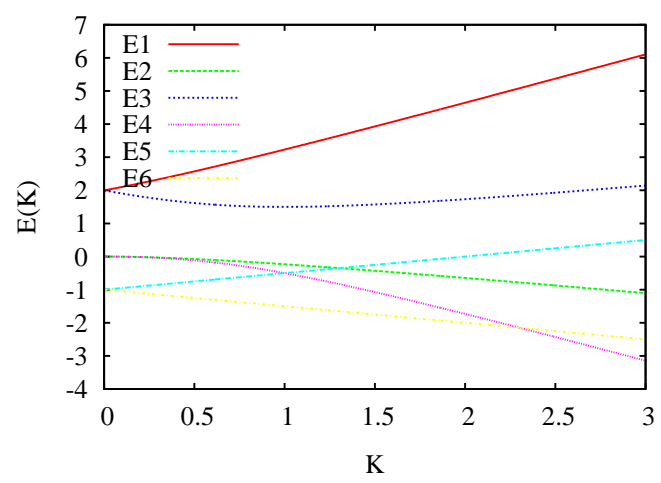

Figure 2. Energy levels of symmetric $\triangle$-type configuration Hamiltonian $H_{0} . E(K)$ are plotted in $J=1$ units

shape, shown in Fig. 1 1 c', the energy gap between low-lying negative energy states and the excited states can be effectively suppressed by increasing the driving field strength $K$. The energy spectrum of the Hamiltonian $H_{0}$ with $J_{01}=J_{02}=J, J_{12}=0$ is shown in Fig. 3. If the system of coupled qubits interacts to a heat bath of phonons, an initial quantum state of such system, prepared as a linear superposition of quantum states of computational basis, will 


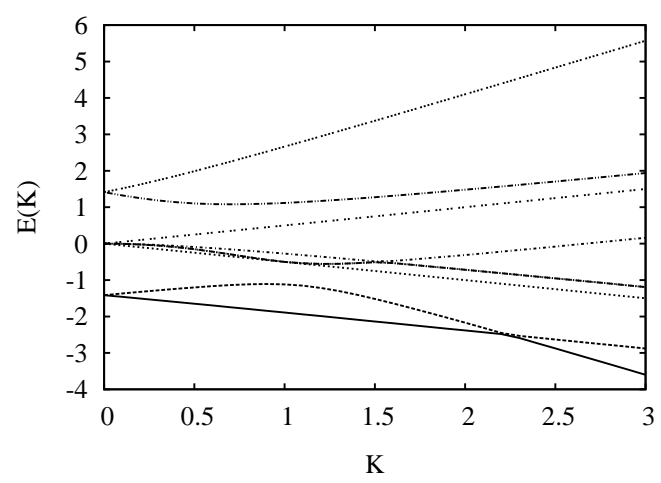

Figure 3. Energy levels of $\Lambda$-type configuration. $E(K)$ are plotted in $J=1$ units

decohere into a mixture of quantum states, unless it does not belong to a decoherence-free subspace, i.e., is not subjected to decoherence [18].

The evolution of 3 qubit quantum system is described by the von Neumann equation for the density matrix

$$
\dot{\rho}=-\frac{\imath}{\hbar}[H, \rho]
$$

where $\rho(t)$ is the total density matrix of the system together with its environment, with the initial state decoupling condition $\rho(0)=\rho_{S Y S}(0) \otimes \rho_{E N V}(0)$. The interaction between the system and environment is assumed be weak enough to write the total Hamiltonian in the form $H=H_{S Y S}+H_{E N V}+\lambda H_{I N T}$, where $\lambda$ is a small parameter. The interaction Hamiltonian $H_{I N T}$ is usually assumed to be linear in system coordinates. From the theory of open quantum system [19], we expect that the fluctuations of environment play the same role in quantum neural networks as they do in any Boltzmann machine, and the system density matrix $\rho_{S Y S}(t)$ will evolve to the minimal energy state of the system. The evolution equation for the system density matrix is given by tracing over the environment degrees of freedom

$$
\dot{\rho}_{S Y S}=-\frac{\imath}{\hbar} \operatorname{Tr}_{E N V}[H, \rho]
$$

where $\rho_{S Y S}=\operatorname{Tr}_{E N V} \rho$. The latter equation (5) can be solved either perturbatively, or numerically, using the numerical methods for quasi-adiabatic path integral [20].

The quantitative measures of the "quantumness" of collective states of quantum system are their entanglement and different types of entropy [21].

Following our previous papers studying two dipole-dipole coupled qubits with common 
phonon bath [17, 22], where we have observed that initially symmetric state

$$
\left|e_{3}\right\rangle=\frac{|X 0\rangle+|0 X\rangle}{\sqrt{2}}
$$

is spread into a uniform mixture of three triplet states (in the Bell basis)

$$
\left|e_{1}\right\rangle=\frac{|X X\rangle+|00\rangle}{\sqrt{2}},\left|e_{2}\right\rangle=\frac{|X X\rangle-|00\rangle}{\sqrt{2}},\left|e_{3}\right\rangle
$$

without mixing to a singlet state $\left|e_{4}\right\rangle=\frac{|X 0\rangle-|0 X\rangle}{\sqrt{2}}$ even in the presence of phonon bath.

This separation to be a consequence of the decomposition of direct product of two spin Hilbert spaces into a sum of invariant subspaces $D_{\frac{1}{2}} \otimes D_{\frac{1}{2}}=\underbrace{D_{1}}_{\text {triplet }} \oplus \underbrace{D_{0}}_{\text {singlet }}$.

Based on the $S U(2)$ representation structure of the three spin qubits states

$$
D_{\frac{1}{2}} \otimes D_{\frac{1}{2}} \otimes D_{\frac{1}{2}}=D_{\frac{3}{2}} \oplus 2 D_{\frac{1}{2}}
$$

where the quartet representation $D_{\frac{3}{2}}$ is totally symmetric with respect to all three spins, we can conjecture similar structure of invariant subspaces, surviving at the presence of phonon bath, at least for symmetric coupling between qubits.

Using the standard computational basis $\left(e_{0}=|000\rangle, \ldots, e_{7}=|X X X\rangle\right)$ we can construct the basis for quartet representation $D_{3 / 2}$ :

$$
\begin{aligned}
& f_{\frac{3}{2},+\frac{3}{2}}=\quad|X X X\rangle=\quad e_{7}, \\
& f_{\frac{3}{2},+\frac{1}{2}}=\quad \frac{|0 X X\rangle+|X 0 X\rangle+|X X 0\rangle}{\sqrt{3}}=\frac{e_{3}+e_{5}+e_{6}}{\sqrt{3}}, \\
& f_{\frac{3}{2},-\frac{1}{2}}=\quad \frac{|X 00\rangle+|0 X 0\rangle+|00 X\rangle}{\sqrt{3}}=\quad \frac{e_{4}+e_{2}+e_{1}}{\sqrt{3}}, \\
& f_{\frac{3}{2},-\frac{3}{2}}=\quad|000\rangle=\quad e_{0} .
\end{aligned}
$$

The bases for two remaining $D_{1 / 2}$ representations can be constructed as the set of vectors orthogonal to the $f_{\frac{3}{2},+\frac{1}{2}}$ and $f_{\frac{3}{2},-\frac{1}{2}}$ :

$$
\begin{array}{rrr}
f_{4}=-\sqrt{\frac{2}{3}} e_{3}+\frac{1}{\sqrt{6}} e_{5}+\frac{1}{\sqrt{6}} e_{6}, & f_{6}=-\sqrt{\frac{2}{3}} e_{4}+\frac{1}{\sqrt{6}} e_{2}+\frac{1}{\sqrt{6}} e_{1}, \\
f_{5}=\frac{1}{\sqrt{2}} e_{5}-\frac{1}{\sqrt{2}} e_{6}, & f_{7}=\frac{1}{\sqrt{2}} e_{2}-\frac{1}{\sqrt{2}} e_{1} .
\end{array}
$$

They form two $D_{1 / 2}$ doublets: $\left(f_{5}, f_{7}\right)$ and $\left(f_{4}, f_{6}\right)$. Similar to the Bell basis, the states $e_{0}$ and $e_{7}$ can be symmetrized $f_{0}=\frac{e_{0}+e_{7}}{\sqrt{2}}, f_{1}=\frac{e_{0}-e_{7}}{\sqrt{2}}$. In matrix form the group basis $f_{k}$ is 
related to the computational basis $e_{l}: f_{k}=A_{k l} e_{l}$, where

$$
A=\left(\begin{array}{cccccccc}
\frac{1}{\sqrt{2}} & 0 & 0 & 0 & 0 & 0 & 0 & \frac{1}{\sqrt{2}} \\
\frac{1}{\sqrt{2}} & 0 & 0 & 0 & 0 & 0 & 0 & -\frac{1}{\sqrt{2}} \\
0 & 0 & 0 & \frac{1}{\sqrt{3}} & 0 & \frac{1}{\sqrt{3}} & \frac{1}{\sqrt{3}} & 0 \\
0 & \frac{1}{\sqrt{3}} & \frac{1}{\sqrt{3}} & 0 & \frac{1}{\sqrt{3}} & 0 & 0 & 0 \\
0 & 0 & 0 & -\frac{2}{\sqrt{6}} & 0 & \frac{1}{\sqrt{6}} & \frac{1}{\sqrt{6}} & 0 \\
0 & 0 & 0 & 0 & 0 & \frac{1}{\sqrt{2}} & -\frac{1}{\sqrt{2}} & 0 \\
0 & \frac{1}{\sqrt{6}} & \frac{1}{\sqrt{6}} & 0 & -\frac{2}{\sqrt{6}} & 0 & 0 & 0 \\
0 & -\frac{1}{\sqrt{2}} & \frac{1}{\sqrt{2}} & 0 & 0 & 0 & 0 & 0
\end{array}\right)
$$

We performed numeric simulations with symmetric configuration with density matrix $\hat{\rho}(0)=$ $|f(3 / 2,+1 / 2)\rangle\langle f(3 / 2,+1 / 2)|$ and calculate the evolution of density matrix for the superohmic spectral density of phonon bath, and $T=77 \mathrm{~K}$. In numerical simulations the values

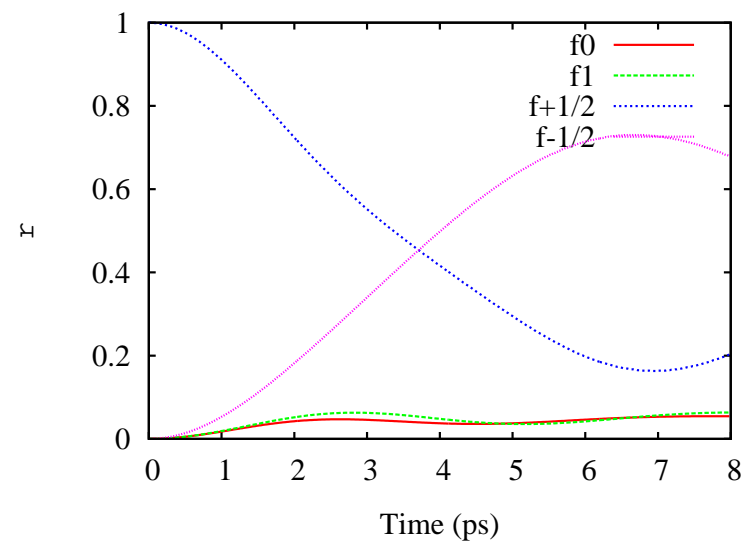

Figure 4. Evolution of diagonal density matrix elements in group basis for $D_{\frac{3}{2}}$ with $\triangle$-type Hamiltonian evaluated by quasi-adiabatic path integral method. The diagonal elements of the orthogonal spaces $D_{1 / 2}$ a kept zero. The nondiagonal elements are not shown.

of the coupling constants $K=0.24, J=0.595$, in accordance to quantum dot sizes and parameters given in [22]. The results of simulations are presented at the phonon bath temperature $T=77 \mathrm{~K}$.

If the interaction Hamiltonian is not symmetric to permutations the initial symmetric state will span the whole basis during the time evolution, as is displayed in Fig. 5 below. Starting from initial state $\rho(0)=\left|f_{7}\right\rangle\left\langle f_{7}\right|$ we have the oscillations between $f_{7}$ and $f_{5}$ 


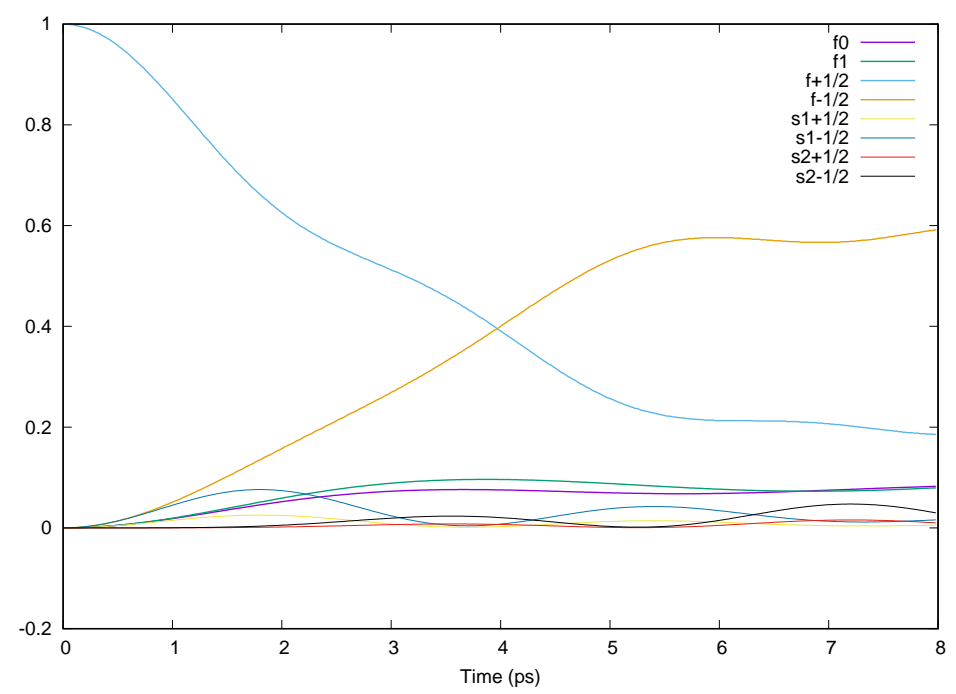

Figure 5. Evolution of diagonal density matrix elements in group basis for $D_{\frac{3}{2}}+2 D_{\frac{1}{2}}$ for $\Lambda$ type Hamiltonian and symmetric $f\left(\frac{3}{2},+\frac{1}{2}\right)$ initial state

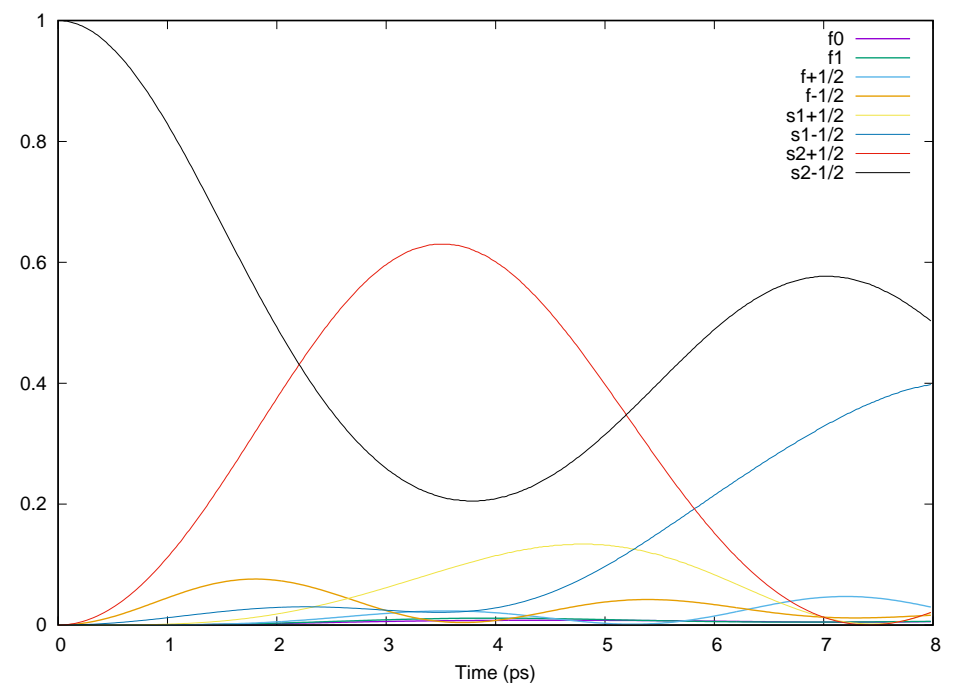

Figure 6. Evolution of diagonal density matrix elements in group basis for $D_{\frac{3}{2}}+2 D_{\frac{1}{2}}$ for $\triangle$ type Hamiltonian and $f_{7}$ initial state

\section{CONCLUSION}

We conclude that the ideas of the decoherence-free subspaces, proposed for singlet states [18], can be exploited for rather general open quantum systems of quantum dots. Depending on the symmetry of connection between the dots, the states of the array can be hold within particular multiplets, the sum of which comprise the space of quantum states of the array. This constrains environment-induced decoherence and provides possibilities for 
building adiabatic quantum computers working at high temperatures.

[1] R. Feynman, Int. J. Theor. Phys. 21, 467 (1982).

[2] D. Deutsch and R. Jozsa, Proc. Roy. Soc. Lond. A 439, 553 (1992).

[3] M. Johnson and et al., Nature 473, 194 (2011).

[4] S. Haykin, Neural Networks (Pearson Education, 1999).

[5] N. G. Berloff, M. Silva, K. Kalinin, A. Askitopoulos, J. D. Topfer, P. Cilibrizzi, W. Langbein, and P. G. Lagoudakis, Nat. Mater. 16, 1120.

[6] Johnson M. W., Amin M. H. S., Gildert S., Lanting T., Hamze F., Dickson N., Harris R., Berkley A. J., Johansson J., Bunyk P., Chapple E. M., Enderud C., Hilton J. P., Karimi K., Ladizinsky E., Ladizinsky N., Oh T., Perminov I., Rich C., Thom M. C., Tolkacheva E., Truncik C. J. S., Uchaikin S., Wang J., Wilson B., and Rose G., Nature 473, 194 (2011).

[7] E. Cohen and B. Tamir, Int. J. Quant. Inf. 12, 1430002 (2014).

[8] M. Schuld, I. Sinayskiy, and F. Petruccione, Quantum Information Processing 13, 2567 (2014).

[9] V. Chavchanidze, Soobshch. AN Gruzinskoi SSR 59, 37 (1970).

[10] F. Beck and J. Eccles, PNAS 89, 11357 (1992).

[11] E. Behrman, L. Nash, J. Steck, V. Chandrashekar, and S. Skinner, Inf. Sci. 128, 257 (2000).

[12] M. Altaisky, N. Kaputkina, and V. Krylov, Physics of Particles and Nuclei 45, 1013 (2014).

[13] M. V. Altaisky, N. N. Zolnikova, N. E. Kaputkina, V. A. Krylov, Y. E. Lozovik, and N. S. Dattani, Applied Physics Letters 108, 103108 (2016).

[14] Y. E. Lozovik and N. E. Kaputkina, Physica Status Solidi B Basic Research 207, 147 (1998).

[15] N. E. Kaputkina and Y. E. Lozovik, Physics of the Solid State 40, 1594 ?1599 (1998).

[16] F. J. Rodríguez, L. Quiroga, and N. F. Johnson, Phys. Rev. B 66, 161302 (2002).

[17] M. Altaisky, N. Zolnikova, N. Kaputkina, V. Krylov, Y. E. Lozovik, and N. Dattani, Photonics and Nanostructures - Fundamentals and Applications 24, 24 (2017).

[18] P. Zanardi and M. Rasetti, Phys. Rev. Lett. 79, 3306 (1997).

[19] R. Feynman and F. Vernon Jr., Ann. Phys. 24, 118 (1963).

[20] N. Makri and D. Makarov, Journal of Chemical Physics 102, 4611 (1995).

[21] C. Bennet, D. DiVincenzo, J. Smolin, and W. Wooters, Phys. Rev. A 54, 3824 (1996). 
[22] M. Altaisky, N. Zolnikova, N. Kaputkina, A. Krylov, Y. E. Lozovik, and N. Dattani, Eur. Phys. J. Web of Conf. 108, 02006 (2016). 